\title{
Chest Pain During Upper Endoscopy
}

\author{
Jae Myung Park, MD
}

Division of Gastroenterology, Department of Internal Medicine, The Catholic University of Korea, School of Medicine, Seoul, Korea

A 75 -year-old male patient visited Seoul St. Mary's Hospital for regular check-up after endoscopic resection of the early gastric cancer. He complained of mild and intermittent dysphagia to solids and liquids for 1 year duration. Frequency of dysphagia increased recently. Previously performed upper endoscopies were reported as normal. After insertion of endoscopy, he complained of severe chest pain. The esophageal mucosa appeared normal but there were severe contractions leading to resistance in advancing the scope in the mid esophagus (Fig. 1). Esophagogram showed a corkscrew appearance on the mid and lower part of the esophagus (Fig. 2). Esophageal manometry disclosed the hypercontractile amplitudes of $365 \mathrm{mmHg}$ (Fig. 3). A normal relaxation of the lower esophageal sphincter ruled out achalasia. Cardiac evaluation revealed nonspecific findings. These findings led to the diagnosis of diffuse esophageal spasm (DES), which was responsible for the chest pain. Chest pain subsided with the treatment of proton pump inhibitor, verapamil and isosorbide mononitrate.

The pain associated with DES are very variable in severity and sometimes can mimic that of angina pectoris. ${ }^{1}$ Upper endoscopy is firstly recommended to detect structural causes of dysphagia and noncardiac chest pain. In most affected patients, the esophagus retains the ability to propagate normal peristaltic contractions. As endoscopy has substantial limitations in assessing abnormal esophageal motility in DES, the intraluminal manometry is usually needed for the diagnosis of increased pressure
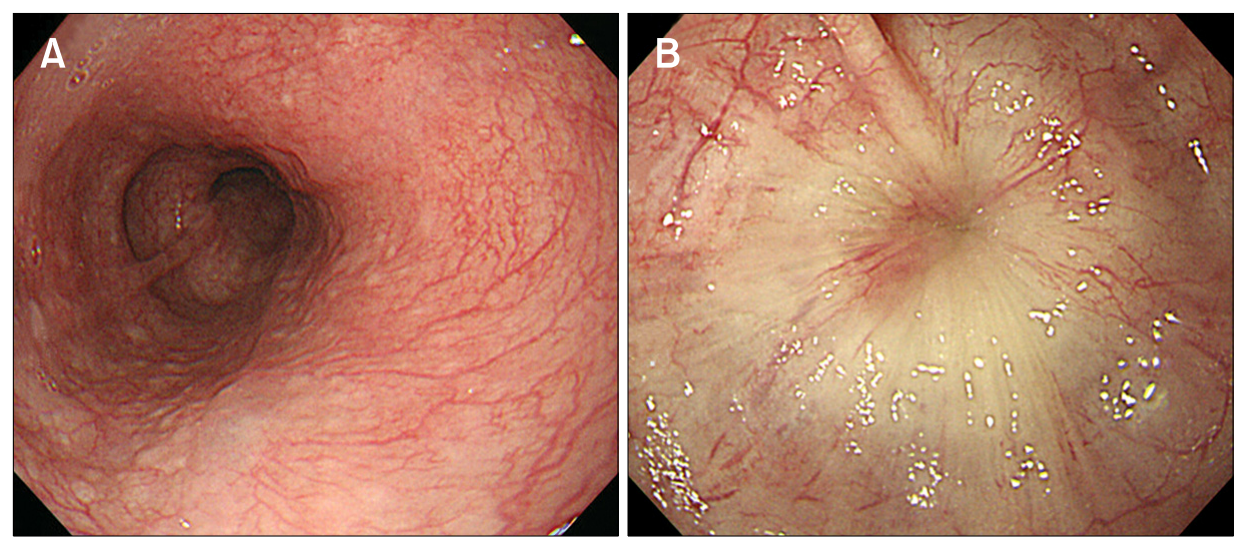

Figure 1. Upper endoscopy before (A) and after (B) the development of chest pain. Powerful contraction of mid esophagus is observed in the figure $1 \mathrm{~B}$.

Received: September 1, 2010 Revised: September 17, 2010 Accepted: September 20, 2010

(c) This is an Open Access article distributed under the terms of the Creative Commons Attribution Non-Commercial License (http://creativecommons. org/licenses/by-nc/3.0) which permits unrestricted non-commercial use, distribution, and reproduction in any medium, provided the original work is properly cited.

*Correspondence: Jae Myung Park, MD

Division of Gastroenterology, Department of Internal Medicine, The Catholic University of Korea, School of Medicine, 505 Banpo-dong, Seocho-gu, Seoul 137-070, Korea

Tel: +82-2-2258-6023, Fax: +82-2-2258-2053, E-mail: parkjerry@catholic.ac.kr

Financial support: None.

Conflicts of interest: None. 


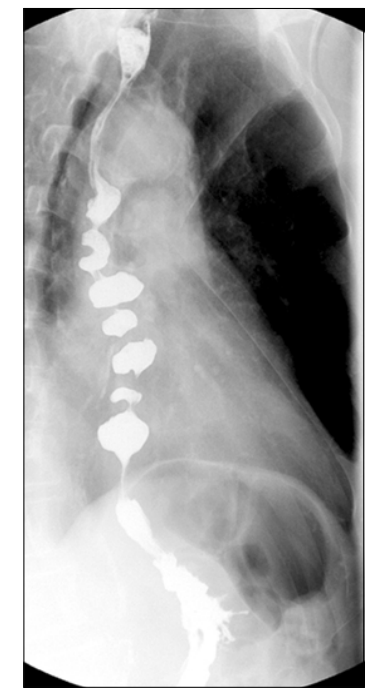

Figure 2. Corkscrew esophagus on barium esophagogram.

of nonperistaltic contractions. ${ }^{2}$

The presented case showed interestingly endoscopic findings strongly suggestive of DES along with compatible radiographic and manometric findings.

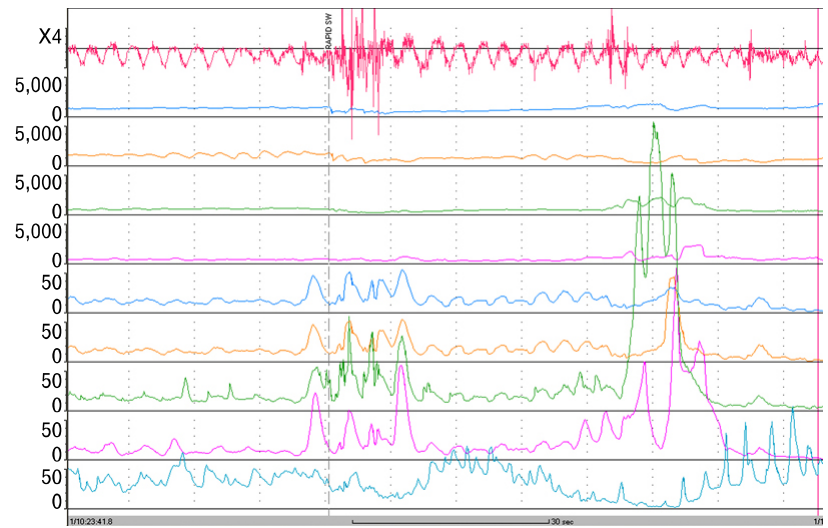

Figure 3. Esophageal manometry shows multiple spontaneous simultaneous contractions. The amplitudes of these contractions are particularly high.

\section{References}

1. Fass R. Evaluation and diagnosis of noncardiac chest pain. Dis Mon 2008;54:627-641.

2. Grübel C, Borovicka J, Schwizer W, Fox M, Hebbard G. Diffuse esophageal spasm. Am J Gastroenterol 2008;103:450-457. 